$\mathbb{P}$ periodica polytechnica

Civil Engineering

$55 / 2(2011) 191+197$

doi: 10.3311/pp.ci.2011-2.11

web: http://www.pp.bme.hu/ci

(c) Periodica Polytechnica 2011

RESEARCH ARTICLE

\section{Examining injury severity in left turning crashes at intersections}

\author{
Yong-gang Wang / Hui Peng / Wen-sen Xiang / Yu-long Pei
}

Received 2010-09-05, revised 2011-05-06, accepted 2011-07-18

\begin{abstract}
In response to the increasing concerns and challenges for most frequently left-turn crashes at intersections, partial proportional odds models, for which some of the beta coefficients vary across variables, are proposed to examine and understand the influence of contributory factors (i.e. human attributes, traffic flow features, roadway geometrics, and environmental factors, etc.) on injury severity involved in left-turn crashes, using the selected 317 crash data over latest 6 years from Xian city. The results show that partial proportional odds model has better performance than general ordered logit or probit probability approach. Specifically, the aged and younger drivers are more prone to cause left-turn crashes, and the increasing effect of trucks involvement, impact points of both vehicles, environmental factors, safety belt usage, alcohol and/or drugs are also significantly associated with higher injury severities, which was underestimated or underreported in previous researches.
\end{abstract}

\section{Keywords}

traffic safety $\cdot$ ordered probability analysis $\cdot$ left-turning collision $\cdot$ crash severity $\cdot$ partial proportional odds model $\cdot$ contributory factor

\section{Yong-gang Wang}

School of Highway, Chang' an University, Middle Section of South 2 Ring Rd., Xi' an 710064, China

e-mail: sdqdwyg@163.com

\section{Hui Peng}

School of Highway, Chang'an University, Middle Section of South 2 Ring Rd., Xi'an 710064, China

e-mail: penghui1963@126.com

\section{Wen-sen Xiang}

Shanghai Municipal Engineering Design General Institute, Shanghai 200030, China

e-mail: xiangwensen0115@163.com

\section{Yu-long Pei}

School of Transportation Science and Engineering, Harbin Institute of Technology, 73 Huanghe Rd., Harbin 150090, China

e-mail: yulongp@263.net

\section{Introduction}

As the most dangerous locations of a roadway network, intersections have a poor safety performance compared with other road location groups [1]. According to the Harbin Traffic Police annual statistics report for 2008, motorcycle crashes constitute about $68.3 \%$ of fatal and about $56.9 \%$ of injury records at intersections controlled by traffic signals [2] and these observations are even more alarming in some hilly cities, such as Qingdao, Dalian, etc. Obviously, intersections are prone to be involved in traffic crashes due to the substantial causal factors, such as vehicular characteristics, driver attributes, pedestrian conflicts, roadway geometry features, environmental factors, etc [3, 4]. How these different factors affect the occurrence of crashes and severities, however, are still not clear, particular left-turn crashes.

There have been considerable research reports on the crash injury severity in the last two decades. A number of researchers have attempted to specify multinomial logit models into quantifying the effects of multiple alternatives (e.g. roadway, traffic, environmental, human and vehicular factors) on intersection related injury severity [5-7]. Chimba and Sando (2011) considered number of lanes, alcohol and drug use, high posted speed limit, curved areas, turning movements, ramps, and driving with no adequate daylight in their multinomial logit and multinomial probit analysis of independent variable effect on each injury severity [8]. Geedipally et al (2010) applied multinomial logit model to predict the proportion of crashes by collision type and to estimate crash counts by collision type and found it produced the best fit even for small sample size [9].

However, ordered nature of severity is inconsistent with the general logit model's assumption that alternatives or variables have independent errors. Hence, nested logit, mixed logit, or probit models can be applied to indentify the severity in terms of levels or grades from a modeling perspective. Young and Liesman (2007) applied the binary logit model to examine whether there was significant correlation between weather station wind data and the likelihood of freight vehicle involved overturning crashes [10]. Tay and Rifaat (2007) applied the ordinal probit model to determine the determinants (i. e., vehicle type, 
road type, collision type, driver's characteristics and time of day, etc) of the severity of intersection crashes using 1992-2002 crash data in Singapore [11].Unfortunately, the assumption of the same coefficients of ordered probability models for different severity levels except for cut-off points is too arbitrary, and determining more parameters than necessary also causes some variables insignificant [12]. Wang and Abdel-Aty (2008) examined the left-turn crash injury severity using partial proportional odds models and reached a consistently better performance than ordered probability models with either logit or probit function [13], where parallel-lines constraint is violated only by one or a few of the included explanatory variables.

In summary, there have been numerous studies investigating crash injury severity. Unfortunately, only limited studies focused on left-turn crash injury severity at intersections specifically, and in previous studies, crash injury features are unable to be associated with contributory factors (i.e., turning movements, signal phase, traffic flow, roadway geometrics, environmental attributes, etc) that depend on sufficient crash data. However, it is difficult to collect crash data or even not available for most intersections. In this study, left-turn crash injury severity is estimated using partial proportional odds models, compared with ordered logit and probit models. Upon the urgent demand, the primary purpose of this research is to specify the effect of specific attributes on crash severity features using the surveyed 2005-2010 intersection crash data in Xi' an city, which enables researchers, engineers and decision-makers to identify and rank intersections for safety performance improvement needs.

\section{Data Collection}

The data is sourced from the Traffic Safety Statistics and Analysis Database developed by Xi' an General Department of Transportation, which consists of data compiled from a sample of police-reported crashes that involve at least one motor vehicle traveling on a traffic road / street and resulting in property damage, injury, or death. A number of crash-related information on crash records, characteristics of the individuals involved, locations of crash, intersection geometric features and traffic control from 2005 to 2010 were obtained for 163 four-legged signalized intersections in Xi' an city. Details include the date of the occurrence, time of day, environment factors, involver age and gender, vehicle characteristics, entering traffic flow, roadway design attributes, and severity level in most observed crashes. According to the direction of vehicle movement including straight ahead, making right-turn and making left-turn, left-turn crashes could be considered as the crashes involved the making left turn vehicles.

Of the 1368 collisions at the selected intersections, 391 were left-turn collisions that account for $28.58 \%$ of all observed collisions at the selected locations. The injury severity of each individual involved in the crash record is collected on a five point ordinal scale in increasing of severity: $1=$ no injury, $2=$ possible injury, 3 = non-incapacitating injury, 4 = incapacitating in- jury, and $5=$ fatal injury [14]. Of the total 391 left turn crashes, $1.17 \%$ crashes are classified as fatal crashes, $52.63 \%$ are classified as injury crashes that at least one person was injured and the rest of them are property damage over CNY 5000 or no injury crashes.

Two approaches were used to select approximate variables for the severity estimation model. The first was to reference previous studies and find these similar reported variables [15, 16]. The second was to focus on local requirement and determine the specific variables that might have significant influence on injury crashes. It should be noted that some important variables such as AADT, intersection width, median exists were not chosen here because of the missing of necessary message in original crash reports. Therefore, 317 observations were finally explored to develop models retaining 9 variables in categories and the further descriptive lists of the corresponding variables were in Table 1 including type of vehicle, fault, crash geometry, safety belt equipment, light condition, age, weather, etc.

\section{Model Specification}

This study aims to evaluate the associations between driver injury severity and possible contributory factors. Since the severity of a crash is recorded in simple ordinal patterns such as fatal, injury and non-injury crashes, thus the ordered probit or logit model could be used to measure the crash involved severity because these approaches yield efficient and consistent estimates [17].

If the crash severity could be considered as an ordinal variable having $m$ categories, Let $j(j=1,2, \ldots, m-1)$ be an index to describe a severity level. The index $j$ for severity level, for example, may take values of "no injury" $(j=1)$, "possible injury" $(j=2)$, "non-incapacitating injury" $(j=3)$, "incapacitating injury" $(j=4)$, and "fatal injury" $(j=5)$. Therefore, the probability of crash $i$ having a severity level $j$ will be specified through an ordered logit model (OLM) [18] as:

$$
\begin{gathered}
P\left(Y_{i}>j\right)=g\left(X_{i} \beta^{\prime}\right)=\frac{\exp \left(X_{i} \beta^{\prime}-\tau_{j}\right)}{1+\exp \left(X_{i} \beta^{\prime}-\tau_{j}\right)}, \\
j=1,2, \cdots, m-1
\end{gathered}
$$

where $X_{i}$ is a $k \times 1$ vector of observed explanatory variables of crash $i, \beta$ is a $k \times 1$ vector of under-determined regression parameters. The model parameter $\beta$ and cut-point $\left(\tau_{j}\right)$ of crash $i$ are estimated by maximum likelihood method [19].

It should be noted that the errors of Eq. (1) is assumed to be homoskedastic. However, such an assumption is violated, then the estimated parameters yield the inconsistency due to the aggregation bias assumption [20]. To overcome the shortcoming of unequal error variances, the heterogeneous choice model (HCM) is proposed [21] as:

$$
\begin{gathered}
P\left(Y_{i}>j\right)=g\left(\frac{X_{i} \beta^{\prime}}{\sigma_{i}}\right)=\frac{\exp \left(\frac{X_{i} \beta^{\prime}}{\sigma_{i}}-\tau_{j}\right)}{1+\exp \left(\frac{X_{i} \beta^{\prime}}{\sigma_{i}}-\tau_{j}\right)}, \\
j=1,2, \cdots, m-1
\end{gathered}
$$


Tab. 1. Driver-injury statistics and percentage distribution. Note: * AADT per lane $>5,000 \mathrm{pcu}$, based on the total number of 317 observations.

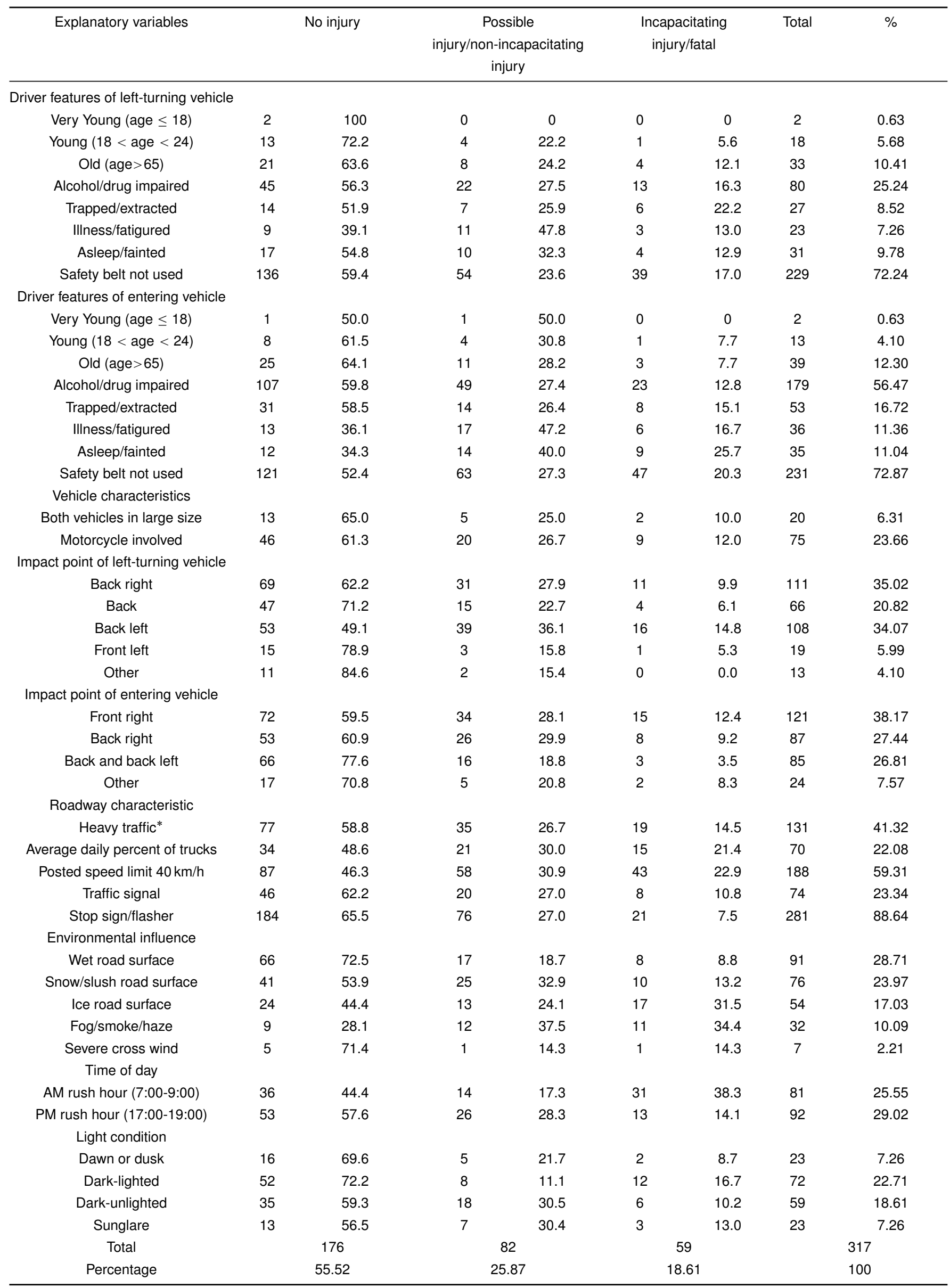


Generally, the OLM regression approach follows the proportional odds / parallel lines assumption and the estimated parameters remain the same for different severity levels. However, this parallel-lines assumption could be violated in many cases. We therefore employ a generalized ordered logit model (GOLM), which would not impose the constraint of parallel regression assumption and allow the effect of the explanatory variables to differ for each category of dependent alternatives [22], which could be specified as:

$$
\begin{gathered}
P\left(Y_{i}>j\right)=g\left(X_{i} \hat{\beta}^{\prime}\right)=\frac{\exp \left(X_{i} \hat{\beta}^{\prime}-\tau_{j}\right)}{1+\exp \left(X_{i} \hat{\beta}^{\prime}-\tau_{j}\right)}, \\
j=1,2, \cdots, m-1
\end{gathered}
$$

Different from the parameter $\beta$ in OLM, vector $\hat{\beta}$ could differ across equations in GOLM. Considering the homoscedasticity assumption might be violated only by one or a few variables, a gamma parameterization of partial proportional odds model with logit function could be proposed [23] as:

$$
P\left(Y_{i}>j\right)=\frac{\exp \left(X_{1 i}^{\prime} \beta^{\prime}+X_{2 i}^{\prime} \beta_{j}^{\prime}-\tau_{j}\right)}{1+\exp \left(X_{1 i}^{\prime} \beta^{\prime}+X_{2 i}^{\prime} \beta_{j}^{\prime}-\tau_{j}\right)}
$$

where the parameter $\beta$ related to variables $X_{1 j}$ would be the same across all equations with respective to different severity level $j$ and the parameter $\beta_{j}$ associated with other variables $X_{2 j}$ would vary across some severity level $j$. In the model, each explanatory variable has one $\beta$ coefficient, $k-2 \beta_{j}$ coefficients, where $k$ is 9 as the number of alternatives. There are $k-1 \tau$ coefficients reflecting cut-off points.

\section{Estimation Results and Analysis}

This study analyzed police-reported intersection crashes from the city of Xi' an, China, from 2005 to 2010, involving only left turning observations. This yields a total of 317 samples. OLM, HCM, GOLM approaches and partial proportional odds model with logit function for both unsignalized intersections and signalized intersections were developed for comparison in case of the quantitative estimation of total left-turn crash injury severity using the proposed nine variables reported in Table 3 which includes some goodness-of-it statistics as well, such as loglikelihood at zero, log-likelihood at convergence, and Akaike information criterion "AIC".

GOLM approach has better performance than the ordered logit model and probit one due to the largest Pseudo $R^{2}(0.0285)$ and the smallest AIC (1063.67). Parallel-lines assumption for each variable was tested through Wald tests to check whether its coefficients differ across injury levels. The variables, safety belt / alcohol involved and environmental influence, are identified to be against the parallel-lines assumption ( $p$-value $<0.01)$. The $p$-values of the Wald test derived from other variables satisfy the parallel-lines assumption for the final model [13].
Traffic flow related variables (i. g., traffic volume, traffic composition, driving behavior, vehicle features, etc.) are identified to be among the most significant factors for left turning crash occurrence. In this study, the effects of different forms of traffic flow on crash injury severity are checked including driver features of left-turning vehicle, driver features of entering vehicle, vehicle characteristics, impact point of left-turning vehicle, impact point of entering vehicle. The results show that very young drivers tend to be involved in more severe crash (Coef. < 0.05 ; $p$-value $<0.01)$, specifically during the rush hour and under the foggy / rainy driving environment. From the total crash data, more than 60 percent are left-turning vehicles at-fault, such as unsafe turning, primarily turning with insufficient headway. On the other hand, more opposing through traffic also causes less time and space for left-turn vehicles due to shorter gaps and therefore cause crashes between two vehicles.

Among the roadway features, average daily percent of trucks is identified to be significant (Coef. $<0.04$; $p$-value $<0.04$ ). Too more trucks will bring poor visibility and decreased capacity of overall intersection and positive restriction of trucks would be better able to provide driving visibility for both drivers and then mitigate the occurrence of left turning crashes as well as lower crash severity. Driver's age also has significant effect on crash injury severity. Compared to middle aged drivers, older than 65 people and young people of age group 18-24 are both more likely to be involved in left-turn crashes due to weak physical conditions and poor driving experiences and very young people are more prone to be get severe injuries and fatalities, which is consistent with previous studies [12,13].

Of the crash related variables, the points of impact of both left turning vehicles and entering vehicles affect crash severity significantly. Coefficients of the factors back right, back, back left, front left are all negative, which indicate that the front of both involved vehicles is the most dangerous impact point, due to the greater colliding forces translated from excessive speed [13]. The difference of variable speeds of both vehicles was marginally significant to affect crash injury and seat-belt use would relieve automatically shock in collision and reduce crash severity to a greater extent (Coef. $<-0.1 ; p$-value $<0.03$ ).

\section{Conclusions}

A large number of previous studies have applied orderedresponse logit / probit models to examine injury severity in relation to human factors, vehicle attributes, and roadway geometrics and environmental effects. These approaches have implicitly assumed the model's independent errors for alternatives that are actually inconsistent with the fact that crash injuries are ordered. The parallel-lines or proportional odds assumption is generally too restricting as well and it may be violated only by one or a few of input variables.

Different from other crash patterns, left-turn crash has its own conflicting features and possibly other specific related factors. This study attempts to estimate the effects of the parameters or 
Tab. 2. Driver-injury severity estimation for left turning crashes. Note: dash (-) indicates data not applicable or unavailable.

\begin{tabular}{|c|c|c|c|c|c|c|}
\hline \multirow{2}{*}{$\begin{array}{c}\text { Variables } \\
\text { Beta }\end{array}$} & \multicolumn{2}{|c|}{ OLM } & \multicolumn{2}{|c|}{$\mathrm{HCM}$} & \multicolumn{2}{|c|}{ GOLM } \\
\hline & Coef. & S.E. & Coef. & S.E. & Coef. & S.E. \\
\hline \multicolumn{7}{|l|}{ Driver features of left-turning vehicle } \\
\hline Very Young (age $\leq 18$ ) & 0.0471 & 0.0036 & 0.0463 & 0.0036 & 0.0411 & 0.0036 \\
\hline Young $(18<$ age < 24$)$ & -0.1706 & 0.7812 & -0.1726 & 0.7739 & -0.1633 & 0.7667 \\
\hline Old (age >65) & -0.2548 & 0.0883 & -0.2601 & 0.0875 & -0.2409 & 0.0867 \\
\hline Alcohol/drug impaired & -0.5013 & 0.2601 & -0.4936 & 0.2577 & -0.5098 & 0.2553 \\
\hline Trapped/extracted & 0.2145 & 0.1837 & 0.2126 & 0.1820 & 0.2073 & 0.1803 \\
\hline Illness/fatigured & -0.1831 & 0.0784 & -0.1872 & 0.0777 & -0.1786 & 0.0770 \\
\hline Asleep/fainted & -0.0726 & 0.2917 & -0.1839 & 0.2891 & 0.0711 & 0.3364 \\
\hline Safety belt not used & 0.2503 & 0.0273 & -0.0763 & 0.0269 & -0.1396 & 0.0266 \\
\hline \multicolumn{7}{|l|}{ Driver features of entering vehicle } \\
\hline Very Young (age $\leq 18$ ) & 0.0487 & 0.0107 & 0.0430 & 0.0106 & 0.0481 & 0.0105 \\
\hline Young $(18<$ age < 24$)$ & -0.1583 & 0.7347 & -0.1601 & 0.7279 & -0.1515 & 0.7211 \\
\hline Old (age >65) & -0.2064 & 0.0819 & -0.2513 & 0.0811 & 0.2235 & 0.2967 \\
\hline Alcohol/drug impaired & -0.4851 & 0.2413 & -0.4579 & 0.2391 & -0.4729 & 0.3369 \\
\hline Trapped/extracted & 0.1990 & 0.1904 & -0.1972 & 0.1886 & -0.1923 & 0.1868 \\
\hline IIIness/fatigured & -0.1699 & 0.0727 & -0.1837 & 0.0720 & -0.1657 & 0.0713 \\
\hline Asleep/fainted & 0.1674 & 0.2706 & -0.1706 & 0.2681 & -0.0660 & 0.2956 \\
\hline Safety belt not used & -0.2322 & 0.0253 & -0.1908 & 0.0251 & -0.1295 & 0.0249 \\
\hline \multicolumn{7}{|l|}{ Vehicle characteristics } \\
\hline Both vehicles in large size & -1.4987 & 0.2137 & -1.4744 & 0.2164 & -1.4794 & 0.1102 \\
\hline Motorcycle involved & -0.9239 & 0.3083 & -1.0082 & 0.3071 & 1.5281 & 0.4204 \\
\hline \multicolumn{7}{|l|}{ Impact point of left-turning vehicle } \\
\hline Back right & -0.2087 & 0.0748 & -0.1977 & 0.0708 & -0.2170 & 0.0777 \\
\hline Back & -0.1762 & 0.0822 & -0.1869 & 0.0779 & -0.2052 & 0.0855 \\
\hline Back left & -0.2596 & 0.0764 & -0.2649 & 0.0724 & -0.2908 & 0.0795 \\
\hline Front left & -0.6635 & 0.2703 & -0.6884 & 0.2560 & -0.7557 & 0.2810 \\
\hline Other & 0.2143 & 0.1928 & 0.1630 & 0.1826 & -0.1789 & 0.2005 \\
\hline \multicolumn{7}{|l|}{ Impact point of entering vehicle } \\
\hline Front right & -1.1762 & 0.1135 & -1.1098 & 0.1132 & -1.2183 & 0.1121 \\
\hline Back right & -1.6776 & 0.0791 & -1.5417 & 0.0791 & -1.6925 & 0.0776 \\
\hline Back and back left & -0.8709 & 0.2647 & -0.8561 & 0.2647 & -0.9398 & 0.2617 \\
\hline Other & 0.1281 & 0.0623 & 0.1106 & 0.0623 & 0.1214 & 0.0609 \\
\hline \multicolumn{7}{|l|}{ Roadway characteristic } \\
\hline Heavy traffic & 0.6137 & 0.1046 & 0.7208 & 0.1041 & 0.7345 & 0.1039 \\
\hline Average daily percent of trucks & -0.4813 & 0.0374 & -0.4275 & 0.0284 & -0.4223 & 0.0261 \\
\hline Posted speed limit 40 km/h & -1.0471 & 0.0747 & -0.9741 & 0.0733 & -0.9622 & 0.0725 \\
\hline Traffic signal & -1.6109 & 0.0651 & -1.5093 & 0.0617 & -1.1336 & 0.0616 \\
\hline Stop sign/flasher & 0.9235 & 0.2833 & 0.9346 & 0.2568 & 1.1232 & 0.2607 \\
\hline \multicolumn{7}{|l|}{ Environmental influence } \\
\hline Wet road surface & -0.3805 & 0.3598 & -0.3976 & 0.3642 & -0.4048 & 0.3962 \\
\hline Snow/slush road surface & 0.7342 & 0.3524 & 0.7431 & 0.3568 & 0.7523 & 0.3871 \\
\hline Ice road surface & -0.5303 & 0.6430 & -0.5369 & 0.6509 & -0.5435 & 0.7083 \\
\hline Fog/smoke/haze & -0.1335 & 0.4523 & -0.1351 & 0.4579 & -0.1368 & 0.4966 \\
\hline $\begin{array}{l}\text { Severe cross wind } \\
\text { Time of day }\end{array}$ & -0.4807 & 0.3758 & -0.4866 & 0.3804 & -0.4926 & 0.4135 \\
\hline AM rush hour (7:00-9:00) & -0.4089 & 0.1337 & -0.4136 & 0.1029 & -0.4379 & 0.0933 \\
\hline PM rush hour (17:00-19:00) & -0.1293 & 0.1158 & -0.1308 & 0.1086 & -0.1536 & 0.1017 \\
\hline \multicolumn{7}{|l|}{ Light condition } \\
\hline Dawn or dusk & -1.3651 & 0.4275 & -1.3336 & 0.4162 & -1.3028 & 0.4068 \\
\hline Dark-lighted & -1.0037 & 0.3311 & -0.9805 & 0.3268 & -0.9579 & 0.3207 \\
\hline Dark-unlighted & 0.4309 & 0.4637 & 0.4209 & 0.4597 & 0.4112 & 0.4515 \\
\hline Sunglare & 0.2298 & 0.6872 & 0.2245 & 0.6804 & 0.2193 & 0.6933 \\
\hline
\end{tabular}


Tab. 3. Driver-injury severity estimation for left turning crashes. Note: dash (-) indicates data not applicable or unavailable.

\begin{tabular}{cccccccc}
\hline Variables & \multicolumn{2}{c}{ OLM } & & HCM & & \multicolumn{2}{c}{ GOLM } \\
\cline { 2 - 5 } Gamma_2 & & & & & & \\
Safety belt / alcohol involved vs. No & - & - & - & - & 0.3275 & 2.4617 \\
Gamma_3 & & & & & & \\
Safety belt / alcohol involved vs. No & - & - & - & - & 0.5163 & 2.7704 \\
Gamma_4 & & & & & & \\
Safety belt / alcohol involved vs. No & - & - & - & - & 0.8819 & 3.1386 \\
Alpha & & & & & & \\
Constant 1 & -0.4448 & - & -0.3874 & - & -0.3082 & 0.2073 \\
Constant 2 & 0.6154 & - & 0.5463 & - & 0.3679 & 0.2498 \\
Constant 3 & 2.2429 & - & 1.8347 & - & 1.3292 & 0.2249 \\
Constant 4 & 5.0396 & - & 3.9703 & - & 2.6953 & 0.2188 \\
Summary Statistics & & & & & & \\
Number of observations & 317 & & 317 & & 317 & \\
Log likelihood at convergence & -498.72 & -486.36 & -471.84 \\
AIC & 1081.25 & 1070.18 & 1063.67 \\
Pseudo $R^{2}$ & 0.0207 & 0.0226 & 0.0285 \\
\hline
\end{tabular}

variables on crash severity, and also compares parametric insights on key roadway geometrics, vehicle, environmental and driver related factors through the application of OLM, HCM, GOLM and partial proportional odds model with logit function approaches when accounting for injury study, aiming at providing reasonable basis to potentially reduce left turning injury severity through advancing the state-of-the-art of injury mitigation strategy, facility manufacturing and policy-making.

Left-turn crashes are located to the crash sites where they occur, which enable to specify the effect of contributory factors on injury severity. The partial proportional odds model has better performance for left-turn crashes, compared with ordered logit or probit model. By using three proposed statistical models, the interpretation of 9 group variables yields greater insight concerning contributing effects of included variables on injury severity. For example, it reveals the aged or younger drivers are more likely to cause left-turn crashes and percentage of trucks involvement also affects the crash occurrence frequency strongly and significantly and increases the injury severity as well. Meanwhile, the points of impact of both vehicles, environmental conditions, safety belt usage, alcohol and/or drugs are found to be strongly associated with higher severities, and all these risk factors should be considered strategically in any future traffic management.

This study is the first step in the statistical estimation of the size of variables affecting injury severity in left-turn crashes. Similar to most previous studies, this approach also has some limitations, such as the fact that small sample data from a single Xi' an city database in Western China, the fact that limited available types of traffic flow related variables from the database, and the fact that traditional statistical models are compared. Future studies with multiple data from different databases and more comprehensive analysis models and methods are strongly encouraged and conducted to confirm the efficiency of the estima- tor for large-scale analysis for left-turn crashes.

\section{Acknowledgement}

This project was supported by the Special Fund for the Basic Scientific Research of Central Colleges, Chang'an University (No. CHD2010JC019). Here the funding and helps from our industry partner, Xi' an General Traffic Policy Teams, are gratefully acknowledged. We would like to thank, in particular, Prof. K.M. Chen from Chang' an University, for suggesting this research problem and the valuable advice he has given.

\section{References}

1 Oosthuizen P, Nienaber $\mathbf{H}$, The status of talent management in the South African consulting civil engineering industry in 2008: a survey, Journal of the South African Institution of Civil Engineering 52 (2010), no. 2, 41-47.

2 Wang Y G, Bai H, Xiang W S, Traffic safety performance assessment and multivariate treatments for intersection locations, The Baltic Journal of Road and Bridge Engineering 6 (2011), no. 1, 30-38.

3 Abdel-Aty M, Salkapuram H, Lee C, Brady P A, A simplistic, practical approach to identify traffic crash profiles at signalized intersections, ITE Journal 76 (2006), no. 4, 28-33.

4 Pulugurtha S S, Sambhara V R, Pedestrian crash estimation models for signalized intersections, Accident Analysis and Prevention 43 (2011), no. 1, 439-446.

5 Xie Y C, Zhang Y L, Liang F M, Crash injury severity analysis using Bayesian ordered probit models, Journal of Transportation Engineering 135 (2009), no. 1, 18-25.

6 Rana T A, Sikder S, Pinjari A R, Copula-based method for addressing endogeneity in models of severity of traffic crash injuries: Application to twovehicle crashes, Transportation Research Record (2010), no. 2147, 75-87.

7 Kim J K, Ulfarsson G F, Shankar V N, Kim S, Age and pedestrian injury severity in motor-vehicle crashes: a heteroskedastic logit analysis, Accident Analysis and Prevention 40 (2008), no. 5, 1695-1702.

8 Chimba D, Sando T, Multinomial probability assessment of motorcycle injury severities, Advances in Transportation Studies (2010), no. 21, 73-80.

9 Geedipally S R, Patil S, Lord D, Examination of methods to estimate crash counts by collision type, Transportation Research Record (2010), no. 2156, $12-20$.

10 Young R K, Liesman J, Estimating the relationship between measured wind speed and overturning truck crashes using a binary logit model, Accident Analysis and Prevention 39 (2007), no. 3, 574-580. 
11 Tay R, Rifaat S M, Factors contributing to the severity of intersection crashes, Journal of Advanced Transportation 41 (2007), no. 3, 244-265.

12 Quddus M A, Noland R B, Chin H C, An analysis of motorcycle injury and vehicle damage severity using ordered probit models, Journal of Safety Research 33 (2002), no. 3, 445-462.

13 Wang X S, Abdel-Aty M, Analysis of left-turn crash injury severity by conflicting pattern using partial proportional odds models, Accident Analysis and Prevention 40 (2008), no. 5, 1674-1682.

14 Tollazzi T, Rencelj M, Rodosek V, Zalar B, Traffic safety of older drivers in various types of road intersections, Promet - Traffic \& Transportation 22 (2010), no. 3, 194-201.

15 Kornelija R, Model for the substantiation of road safety improvement measures on the roads of Lithuania, The Baltic Journal of Road and Bridge Engineering 4 (2010), no. 2, 116-123.

16 Schuchmann G, Road network vulnerability-evaluation of measures in ranking damages and developments, Periodica Polytechnica - Civil Engineering 54 (2010), no. 1, 61-65.

17 Nassiri $\mathbf{H}$, Edrissi A, Alibabai $\mathbf{H}$, Estimation of the logit model for the online contraflow problem, Transport 25 (2010), no. 4, 433-441.

18 Yannis G, Antoniou C, Vardaki S, Kanellaidis G, Older drivers' perception and acceptance of in-vehicle devices for traffic safety and traffic efficiency, Journal of Transportation Engineering 136 (2010), no. 5, 472-479.

19 Arentze T A, Ettema D, Timmermans H J P, Estimating a model of $d y$ namic activity generation based on one-day observations: method and results, Transportation Research Part B: Methodological 45 (2011), no. 2, 447460.

20 Quddus M A, Wang C, Ison S G, Road traffic congestion and crash severity: econometric analysis using ordered response models, Journal of Transportation Engineering 136 (2010), no. 5, 424-435.

21 Srinivasan K K, Naidu G M, Sutrala T, Heterogeneous decision rule model of mode choice incorporating utility maximization and disutility minimization, Transportation Research Record (2009), no. 2132, 59-68.

22 Srinivasan K K, Injury severity analysis with variable and correlated thresholds: ordered mixed logit formulation, Transportation Research Record (2002), no. 1784, 132-142.

23 Tien F F, Fu T T, The correlates of the digital divide and their impact on college student learning, Computers and Education 50 (2008), no. 1, 421436. 